\title{
Family Medicine Specialist S' Opinions on COVID19 Active Screening and Surveillance
}

\section{Rawa Al Ameri* \\ Family Medicine Specialist, Al-Mustansiriyah University, Iraq}

*Corresponding author: Rawa Al-Ameri, Family Medicine Specialist, AlMustansiriyah University, Iraq, Email: dr_rj1983@yahoo.com

\section{Research Article}

Volume 3 Issue 4

Received Date: June 19, 2020

Published Date: July 15, 2020

DOI: $10.23880 /$ jqhe-16000171

\section{Abstract}

Introduction: COVID19 pandemic forced ministries of health across the world to invent additional measures for control. Active screening is one of these tools. It includes asking questions, taking temperatures and doing rapid test for COVID19 in persons with risk factors. A team of a family medicine physician, lab worker, and administrative is formed. They visit homes with positive cases, making physical exam and COVID19 rapid test to contacts of the cases.

Subject and Method: An electronic questionnaire is introduced to family physicians specialists in family medical centers in Baghdad. 99 physicians respond to questionnaire for one week. The questionnaire involved two sections; the 1st one asked if the screening surveillance is necessary for COVID19 control from physician s' point of view. The 2 nd section states the reasons of their opinions.

Results: The study included 99 family medical physicians, 56 said yes; screening is necessary to control viral spread while, 43 said no.

Discussion and Conclusions: Active screening required intense efforts by medical team with limited resources and hot weather. Rapid test of COVID19 is screening test so it is not detecting all infected people, including some with clinical disease compatible with COVID-19. The study gives a conclusion that there is a controversy about active screening.

Keywords: COVID19; Infections; Chest X-Ray

\section{Introduction}

COVID19 pandemic forced ministries of health across the world to invent additional measures for control. Active screening is one of these tools. It includes asking questions, taking temperatures and doing rapid test for COVID19 in persons with risk factors. A team of a family medicine physician, lab worker, and administrative is formed. They visit homes with positive cases, making physical exam and COVID19 rapid test to contacts of the cases. Another way of active screening includes screening of entire city as it is done in Al- Hurriya city due to presence of many cases in it [1-3].

Positive screening rapid test cases were sent to hospitals for diagnostic nasopharyngeal smear. Many positive cases showed negative smears. This result gave a possibility of false positive rate of rapid rate. A study showed that the positive predictive value of the active screening was only $19.67 \%$, and the false-positive rate was $80.33 \%$, with a $75 \%$ probability for the false-positive rate [4].

Another study, done on the close contacts of COVID-19 patients, showed that approximately half or even more of the asymptomatic individuals could be false positives.

So the screening could miss most cases as the contacts usually did not develop symptoms yet [5].

In May, cases suddenly raise dramatically after weeks of stability. Many family physicians referred that to screening surveillance that started in the previous month. Many cases from medical field emerged during the screening as this is 
blamed as contributing factor for viral spread that many asymptomatic medical staff did the routine screen in cities
[1].

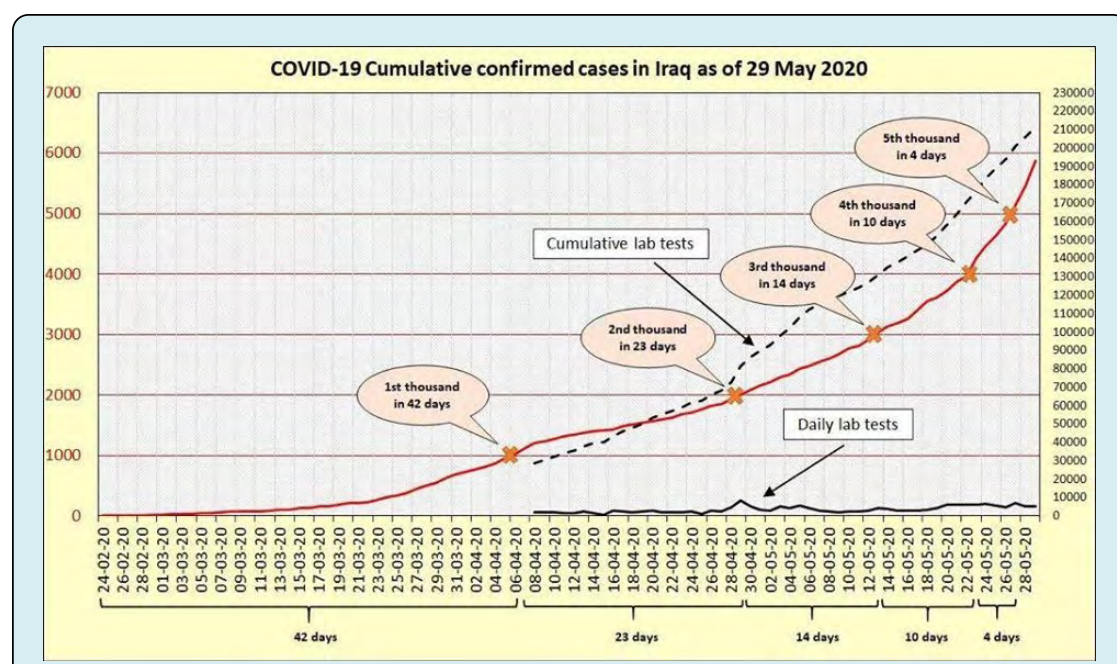

Figure1: COVID19 cumulative confirmed cases during May [1].

\section{Subject and Method}

An electronic questionnaire is introduced to family physicians specialists in family medical centers in Baghdad. 99 physicians respond to questionnaire for one week. The questionnaire involved two sections; the 1st one asked if the screening surveillance is necessary for COVID19 control from physician s' point of view. The $2^{\text {nd }}$ section states the reasons of their opinions.

\section{Results}

The study included 99 family medical physicians, 56 said yes; screening is necessary to control viral spread while, 43 said no.

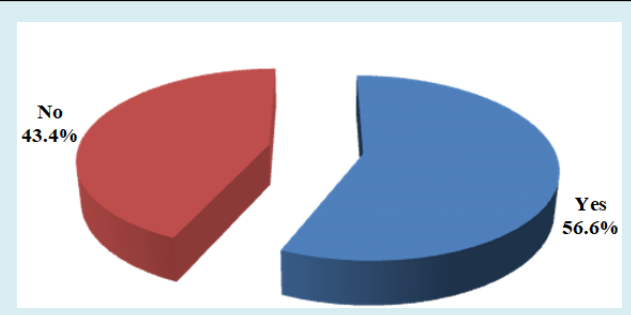

Figure 2: Bar chart showed the percentage of physicians participants.

This study revealed that $48 \quad(85.7 \%)$ family physicians stated that the screening can detect the asymptomatic patients while, $35(81.4 \%)$ physicians blamed it for high infection risk of the screening teams.

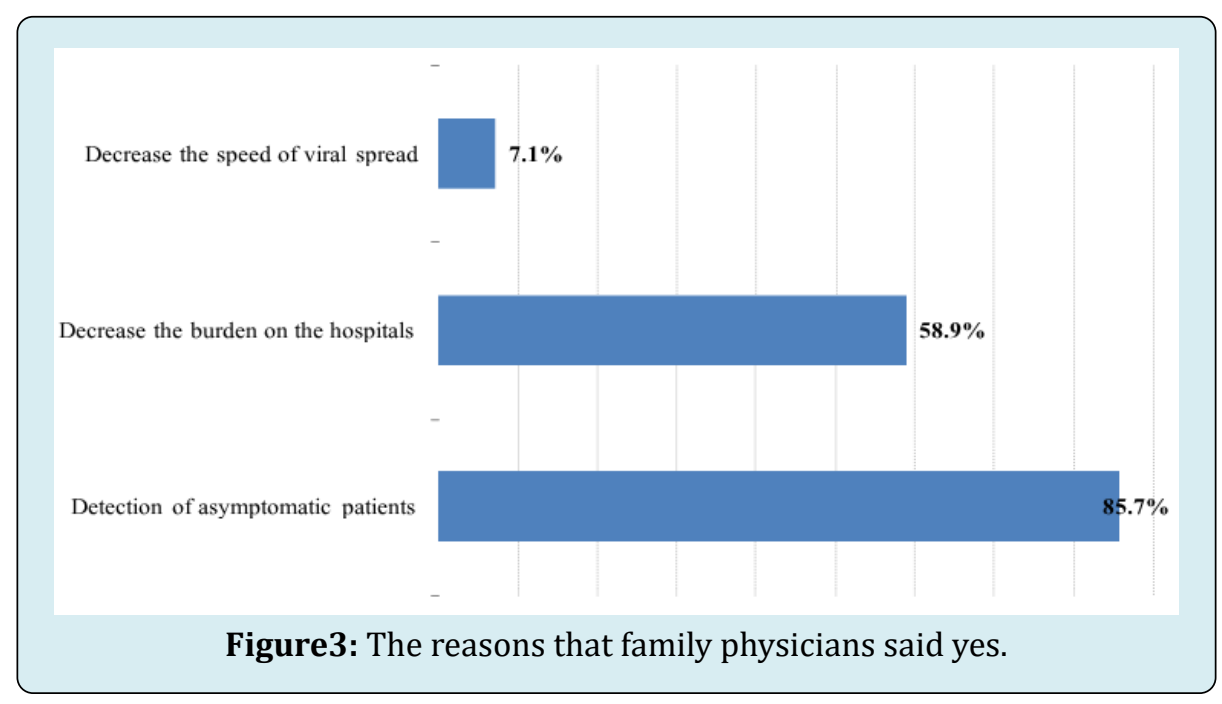




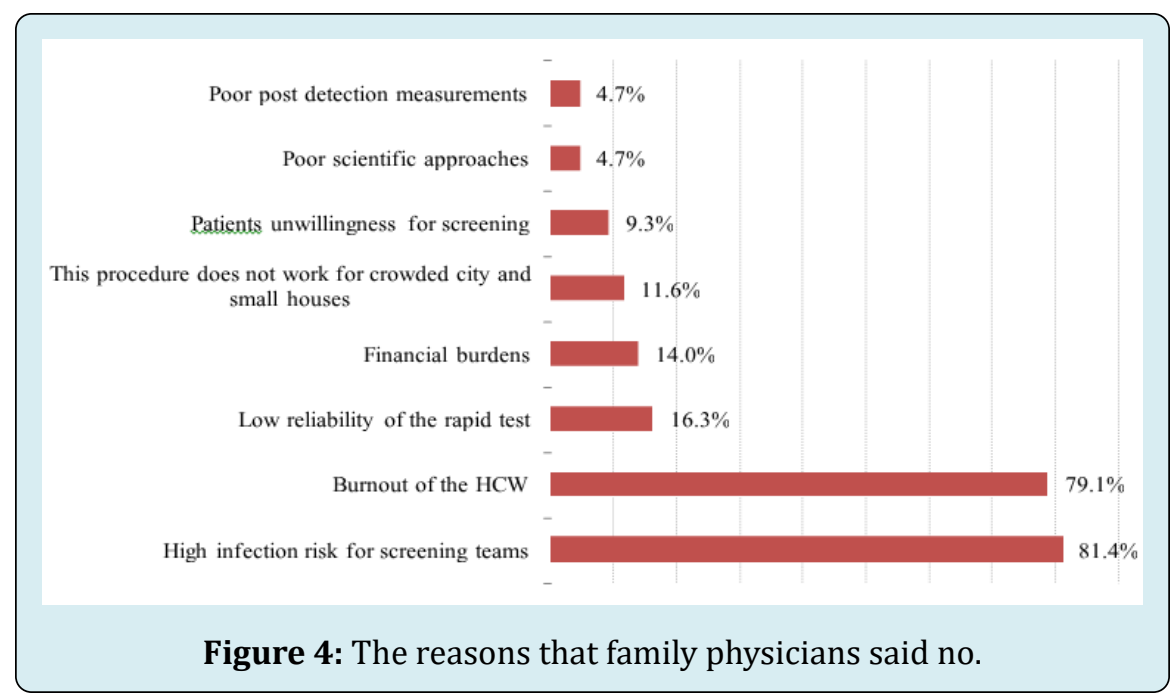

\section{Discussion and Conclusions}

Active screening required intense efforts by medical team with limited resources and hot weather. Studies showed that there are likely many undetected cases because rapid test of COVID19 is screening test so it is not detecting all infected people, including some with clinical disease compatible with COVID-19. Additionally, it will not stop the spread of covid19 but it is a part of a strategy of the World Health Organization of measures for rapid diagnosis and immediate isolation of cases, following and self-isolation of close contacts $[1,6]$.

The current study revealed that 56 family physicians said yes the active screening is necessary in COVID19 control, $85.7 \%$ of them stated that active screening can detect asymptomatic patients. Others opinions were decreasing the hospital burden and limiting viral spread. These physicians may think that early tracking of cases and contacts can offer better prognosis and well isolation to prevent viral spread especially, if it is associated with in home treatment and follow up with referral of only severs cases to hospital.

This opinion would be more applicable if is associated with people commitment of self-isolation and staying home.

The other physicians said no, as $81.4 \%$ of them stated that active screen could increase risk of infection to teams as continuous exposure to cases can increase the viral load and get the infection. This requires making several shifts of teams to avoid exposing the same team to infection, but shortage of numbers of physicians is constant obstacles.

Family medicine specialists mentioned that work burn out is one of other reasons to say no, as $79.1 \%$ of them mentioned that cause. Exhaustion of the physicians that stayed in primary health care due to over work, particularly, in crowded cities as primary health care had large numbers of patients attended it daily.

The study revealed that $16.3 \%$ of family physicians stated that rapid test of COVID19 is not reliable as many negative test showed the disease later. In other hand, positive test showed negative nasopharyngeal smear. Most studies revealed that combinations of clinical symptoms, chest x-ray and CT scan if available gave best results as a study, is done by Ali Narin, et al. exhibited that chest x-ray had $98 \%$ accuracy [7]. Another study revealed that chest x-ray had 96\% sensitivity [8-10].

The study presented that $14 \%$ of family physicians stated that the active screening might cause financial burdens on the health care workers themselves due to lack of resources and on the health system which is unable to compensate the rapid raise in cases numbers.

The study revealed that $11.6 \%$ of physicians stated that the screening procedures might not work in crowded cities with small houses as self- isolation is difficult because many persons live together in small house.

Patient unwillingness for screening is another obstacle, as $9.3 \%$ of physicians identified that cause. Many contacts refused to do screening tests, left the house or did not answer phone calling.

Poor scientific approach is stated by $4.7 \%$ of family physicians. Physicians who are doing the active screen are the same who are doing the routine medical work in family medical center that are dealing with children, elderly, pregnant and other patients without isolation of involved teams. Some family medical centers even did not check their workers by rapid test of covid19. These causes raised the 
suspicion of undetected covid19 cases within the medical field that might spread.

Infection during screening of cities. Others physicians, $4.7 \%$, stated that there is poor post detection measurement. This could be due to increasing number of cases with limited numbers of health care workers.

The study gives a conclusion that there is a controversy about active screening.

\section{References}

1. (2020) novel coronavirus (COVID-19) update from Ministry of Health.

2. (2020) Health Ministry COVID-19 Screening Tool for Long-Term Care Homes and Retirement Homes pp: 2-4.

3. (2020) COVID-19 Guidance: Community Labs and Specimen Collection Centres Active and Passive Screening. Ministry of Health.

4. Zhuang GH, Shen MW, Zeng LX, Mi BB, Chen YF, et al. (2020) Potential false-positive rate among the "asymptomatic infected individuals" in close contacts of COVID-19 patients. Zhonghua Liu Xing Bing Xue Za Zhi
41(4): 485-488.

5. Gostic KM, Gomez ACR, Mummah RO, Kucharski AJ, Lloyd Smith JO, et al. (2020) Estimated effectiveness of symptom and risk screening to prevent the spread of COVID-19. Elife 9: e55570.

6. Marcel S, Christian AL, Richard N, Silvia S, Emma $\mathrm{H}$, et al. (2020) COVID-19 epidemic in Switzerland: On the importance of testing, contact tracing and isolation. Swiss Med Wkly 150: w20225.

7. Narin A, Kaya C, Pamuk Z (2020) Automatic Detection of Coronavirus Disease (COVID-19) Using X-ray Images and Deep Convolutional Neural Networks.

8. Zhang J, Xie Y, Li Y, Shen C, Xia Y (2020) COVID-19 Screening on Chest X-ray Images Using Deep Learning based Anomaly Detection.

9. Moy L, Bluemke D (2020) The Radiology Scientific Expert Panel. Radiology 27: 204005.

10. Ai T, Yang Z, Hou H,Zhan C, Chen C, etal. (2020) Correlation of Chest CT and RT-PCR Testing in Coronavirus Disease 2019 (COVID-19) in China: A Report of 1014 Cases. Radiology 200642. 